Historic, Archive Document

Do not assume content reflects current scientific knowledge, policies, or practices. 



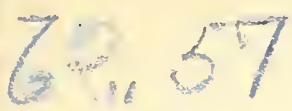

REFERENCES

CITY NATIONAL BANK

AND TRUST Co.

SALEM. N. J.

DUN \& BRADSTREET AGENCIES

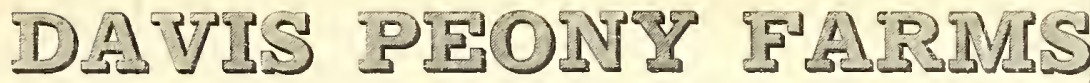

GROWERS OF
E: M. GARRISON, MANAGER

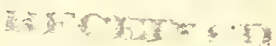

MAR 13 19a औ

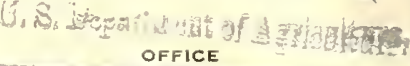

87 WESTBROADWAY

SALEM, N.J.

QUINTON, NEW JERSEY, U. S. A., February 21, 1931

\section{DAHLIA TUBERS AT WHOLESALE}

We list below our offerings for the season 1931. Tubers are true to name, free from disease and field grown. Plants are cultivated by horse drawn machinery, no cut flowers sold from plants. Every tuber with an eye and carefully labeled. Packed in best possible condition.

\begin{tabular}{|c|c|}
\hline Ambassador …............ & $\$ .50$ \\
\hline Amarillo Grande ................ & .75 \\
\hline American Triumph ......... & 3.75 \\
\hline Alex. Pope .................... & 1.00 \\
\hline A. C. Lorbeer ................ & 1.00 \\
\hline Aztec Glory .................... & 2.00 \\
\hline Bashful Giant ................... & .50 \\
\hline Barbara Redfern ........... & 1.00 \\
\hline Big Chief ......................... & 3.00 \\
\hline Charles Stratton ............. & .50 \\
\hline California Superba ... & .50 \\
\hline Champagne & .75 \\
\hline Casper G. Ware & .75 \\
\hline C. B. Githens ....... & .50 \\
\hline City of Trenton ........... & 2.00 \\
\hline Cora Butterworth ....... & 4.00 \\
\hline Elkridge ........................ & 1.00 \\
\hline Elsie Danie!s ............... & .50 \\
\hline Ellinor Vandicvere ........... & .50 \\
\hline Eastern Star ...................... & 1.00 \\
\hline Emma Maric ........... & .50 \\
\hline Eliza Clark Bull .............. & .75 \\
\hline Eagle Rock Beauty ....... & 1.25 \\
\hline Eagle Rock Jewel .............. & 2.00 \\
\hline Eagle Rock Wonder ...... & 10.00 \\
\hline Eagle Rock Gem & 3.00 \\
\hline Edna Ferber .................... & 2.00 \\
\hline El Rey & 5.00 \\
\hline Fordhook Marvel ..... & 1.25 \\
\hline Ft. Monmouth ............... & 2.00 \\
\hline Ft. Washington ........ & 1.50 \\
\hline Flaming Meteor ................. & .50 \\
\hline Grenadier ............................ & .50 \\
\hline Grissette & .50 \\
\hline Giant Ruby ............ & .50 \\
\hline 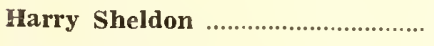 & .50 \\
\hline 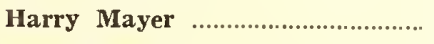 & 1.00 \\
\hline 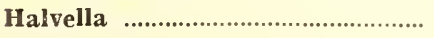 & .50 \\
\hline
\end{tabular}

\begin{tabular}{|c|c|}
\hline His Majesty ........ & 1.00 \\
\hline H. Tribune Elite & 1.00 \\
\hline nsulinde ......................... & .50 \\
\hline I. de Ver Warner & .50 \\
\hline 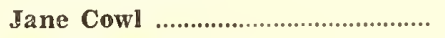 & 1.00 \\
\hline Jersey's Beauty ................................. & .50 \\
\hline Jersey's Mammoth .... & 1.50 \\
\hline Jersey's Jewel .................. & 1.00 \\
\hline Jersey's Sovereign ....... & .50 \\
\hline Jersey's Radiant ........ & .50 \\
\hline Jersey's Queen .................... & .50 \\
\hline Jerscy's Beacon ............... & .50 \\
\hline Jersey's Ideal ................... & 1.00 \\
\hline Jersey's Empress ....... & 1.00 \\
\hline Jersey's Wonder ........ & .75 \\
\hline Jersey's Masterpiece & 2.00 \\
\hline Jersey's Delight ................ & 1.00 \\
\hline Jessie K. Prescott ........................... & .50 \\
\hline Judge Leon McCord .................... & 4.00 \\
\hline Kathlcen Norris .............. & 4.00 \\
\hline Femps Violet Wonder .... & 3.00 \\
\hline Kentucky ........................... & 3.60 \\
\hline King Midas ......... & 3.00 \\
\hline La Grosse Bete ...... & .50 \\
\hline Lavendula ................. & 1.00 \\
\hline Lorelei $\quad . . . . . . . . . . . . .$. & 1.00 \\
\hline My Maryland ............... & 2.00 \\
\hline 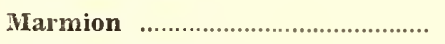 & .75 \\
\hline Margaret Masson ............................... & .50 \\
\hline Mrs. J. T. Scheepers …................... & .50 \\
\hline Mordella & .50 \\
\hline Miss New York ................................ & 1.50 \\
\hline 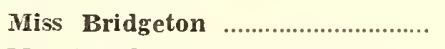 & .50 \\
\hline 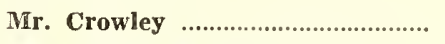 & .50 \\
\hline MIrs. Alfred B. Seal ......................... & 5.00 \\
\hline Mrs. Eleanor Martin ........................ & .75 \\
\hline Mrs. E. L. Linãsay ........................... & .50 \\
\hline Mariposa & .50 \\
\hline
\end{tabular}

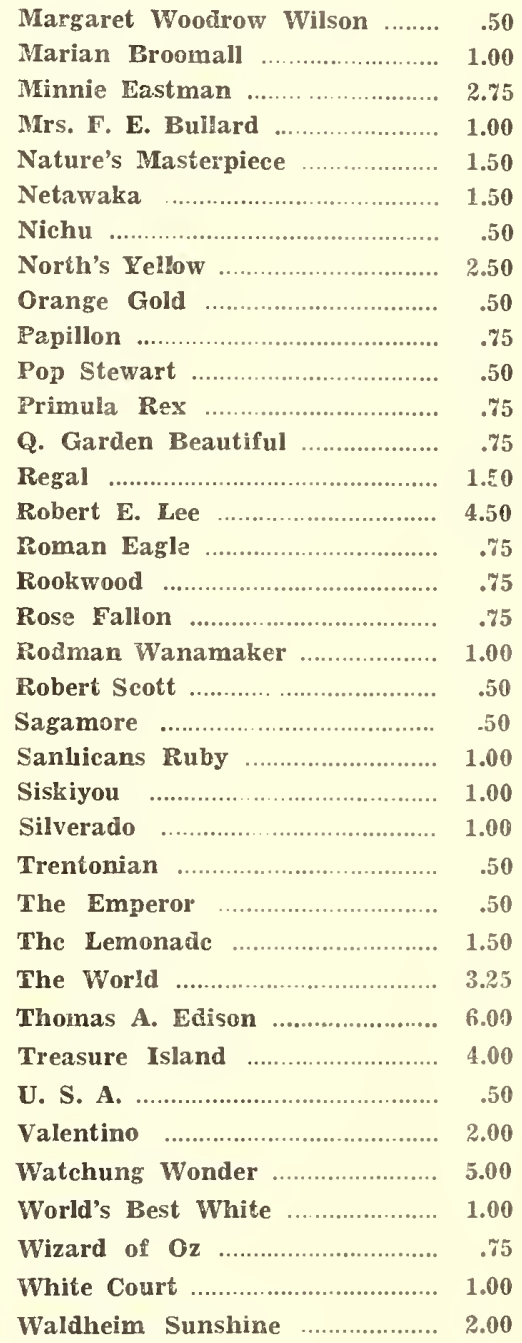

We grow many Dahlias not listed above. Write for prices on any variety you do not see listed. Our terms are cash with the order. If wanted Parcel Post, include postage, otherwise shipments will be sent via Express, collect. 

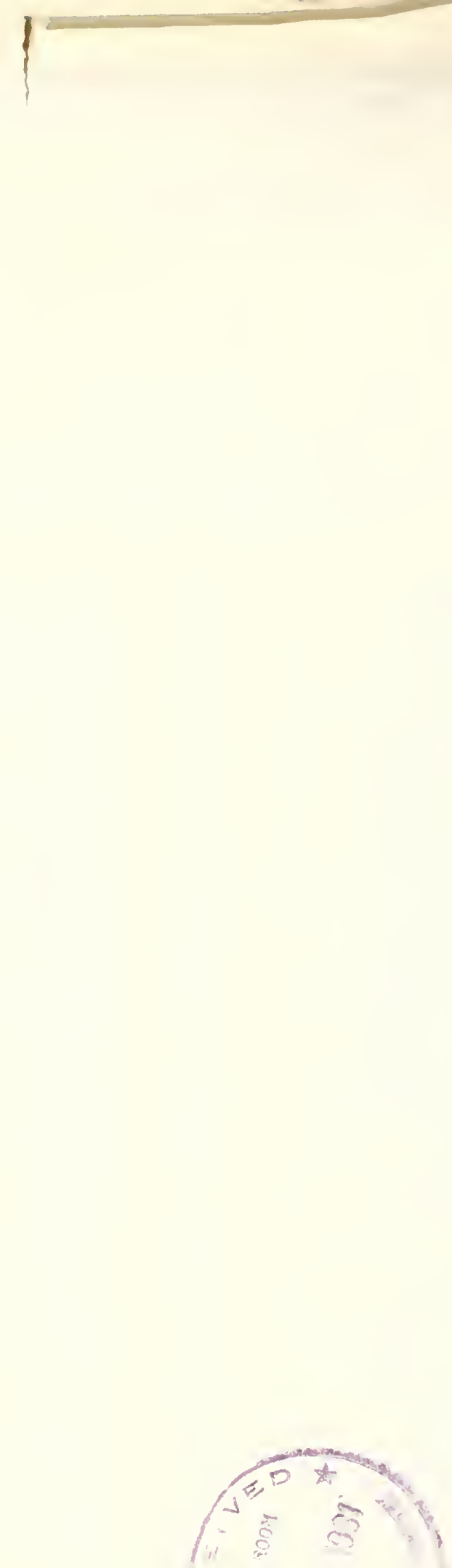